\title{
Granulocyte-Colony Stimulating Factor as Treatment Option in Patients with Recurrent Miscarriage
}

\author{
Claudia Santjohanser $\cdot$ Catherine Knieper $\cdot$ \\ Cordula Franz • Kaino Hirv • Osama Meri • \\ Manfred Schleyer $\cdot$ Wolfgang Würfel $\cdot$ Bettina Toth
}

Received: 11 May 2012/ Accepted: 20 December 2012/Published online: 24 January 2013

(C) L. Hirszfeld Institute of Immunology and Experimental Therapy, Wroclaw, Poland 2013

\begin{abstract}
In 1-5\% of patients during childbearing years recurrent miscarriages $(\mathrm{RM})$ occur. There are established risk factors like anatomical, endocrine and hemostatic disorders as well as immunological changes in the maternal immune system. Nevertheless, further elucidation of the pathogenesis remains a matter of debate. In addition, there are no standardized immunological treatment strategies. Recent studies indicate possible effects of tumor necrosis factor $\alpha$ blocker and granulocyte-colony stimulating factor (G-CSF) concerning live birth rate (LBR) in RM patients. Therefore, we performed a retrospective cohort study in patients undergoing assisted reproductive treatment (ART) with known RM analysing the possible benefits of G-CSF application. From January 2002 to December 2010, 127 patients (199 cylces) with RM (at least 2 early miscarriages) 49 ( 72 cycles) receiving G-CSF and 78 (127 cycles) controls receiving either no medication (subgroup 1) or Cortisone, intravenous immunoglobulins or low molecular weight heparin (subgroup 2) undergoing ART for in vitro fertilisation/intracytoplasmic sperm injection were analysed. G-CSF was administered weekly once (34 Mill) in 11 patients, 38 patients received $2 \times 13$ Mill G-CSF per
\end{abstract}

W. Würfel and B. Toth contributed equally.

C. Santjohanser · O. Meri · M. Schleyer · W. Würfel

Kinderwunsch Centrum München, Munich, Germany

C. Knieper $\cdot$ C. Franz $\cdot$ B. Toth $(\bowtie)$

Department of Gynecological Endocrinology

and Fertility Disorders, University of Heidelberg,

Vossstrasse 9, 69115 Heidelberg, Germany

e-mail: bettina.toth@med.uni-heidelberg.de

K. Hirv

Center for Human Genetics and Laboratory Medicine,

Martinsried, Germany week until the 12th week of gestation. Statistical analysis was performed with SPSS for Windows (19.0), $p<0.05$ significant. The mean age of the study population was $37.3 \pm 4.4$ years (mean \pm standard deviation) and differed not significantly between patients and subgroups. However, the number of early miscarriages was significantly higher in the G-CSF group as compared to the subgroups (G-CSF $2.67 \pm 1.27$, subgroup $10.85 \pm 0.91$, subgroup $20.64 \pm 0.74$ ) and RM patients receiving G-CSF had significantly more often a late embryo transfer (day 5) (G-CSF $36.7 \%$, subgroup $112.1 \%$, subgroup $28.9 \%$ ). The LBR of patients and the subgroups differed significantly (G-CSF $32 \%$, subgroup $113 \%$, subgroup $214 \%$ ). Side effects were present in less than $10 \%$ of patients, consisting of irritation at the injection side, slight leukocytosis, rise of the temperature $\left(<38^{\circ} \mathrm{C}\right)$, mild bone pain and hyperemesis gravidarum. None of the newborn showed any kind of malformations. According to our data, G-CSF seems to be a safe and promising immunological treatment option for RM patients. However, with regard to the retrospective setting and the possible bias of a higher rate of late embryo transfers in the G-CSF group additional studies are needed to further strengthen our results.

Keywords Recurrent miscarriage .

Assisted reproduction - Immunological treatment . G-CSF

\section{Introduction}

Recurrent miscarriage (RM) is defined as more than three miscarriages by the World Health Organization (WHO) (Stirrat 1990). The American Society for Reproductive Medicine defines it as two or more consecutive pregnancy 
losses documented by ultrasound or histopathologic examination (2013). It affects approximately $1-5 \%$ of couples trying to conceive and remains unexplained in about $50 \%$ (Faridi and Agrawal 2011; Toth et al. 2010b). Immunologic processes play a main part during implantation and embryo as well as fetal development (Yang et al. 2010). Most recently, several authors published data on alterations in the humoral and adaptive immune system in RM patients and controls (Hiby et al. 2010; Jin et al. 2011; Toth et al. 2010a; Wang et al. 2010; Yang et al. 2010). Since the discovery of regulatory $\mathrm{T}$ cells (Tregs), the Th1/Th2 paradigm (Jin et al. 2011; Mosmann and Coffman 1989) was challenged towards a more complex model (Kumar et al. 2011; Wang et al. 2010) also including dendritic cells. Dendritic cells present affiliated antigens and are able to induce a switch in the immune answer. Being in the immuntolerant "steady state", they support the physiologic immunoarchitecture of the placenta. When they are exposed to inflammatory stress, they promote effector cells like cytotoxic $\mathrm{T}$ cells to expand and therefore highly contribute to a cytotoxic reaction ( $\mathrm{Scholz}$ et al. 2008; Segerer et al. 2012).

Focussing on the Tregs and Th17 interaction, some aberrations seem to be present in RM patients. Tregs are immunmodulating cells suppressing lymphocytes and are able to produce transforming growth factor $\beta$ and interleukin 10. There is evidence that Treg levels are reduced in RM patients and that a high level of Tregs in the peripheral blood correlates with implantation success after in vitro fertilisation (IVF) (Lee et al. 2012; Zhou et al. 2012). In addition, low circulating $\mathrm{CD} 4{ }^{+} \mathrm{CD} 25^{+}$Foxp $3^{+}$Treg cells seem to predict miscarriage in pregnant women with a history of failure (Winger et al. 2009). T cell subsets like Th17 promote a pro-inflammatory immune reaction and are negatively controlled by Tregs (D'Addio et al. 2011). In the peripheral blood of patients with idiopathic RM, the expression of Th17-positive cells seems to be significantly increased when compared to women with healthy pregnancies (Wang et al. 2010). In addition, the suppressive activity of Tregs towards Th17-positive cells was decreased in RM patients (Wang et al. 2010).

However, the "bench to bedside" process is not finished as there are no standardized procedures to detect immunological disorders in RM patients and accordingly no standardized treatment options to rule out and treat RM patients with immunologic disorders. Even though alterations in natural killer (NK) cells, lymphocytes, T cells or intereleukins can be detected in detail, no gold standards for medical interventions do exist.

So far, immunmodulatory therapies in RM patients include paternal immunization, intravenous immunoglobulins (ivIgG) as well as Cortisone administration. Recent studies indicate a possible role of tumor necrosis factor
(TNF)- $\alpha$ blockers and granulocyte-colony stimulating factor (G-CSF) in RM patients (Scarpellini and Sbracia 2009; Winger et al. 2009).

TNF- $\alpha$ is a pro-inflammatory Th1 cytokine and the ratio of TNF- $\alpha$ producing $\mathrm{T}$ cells seems to be significantly higher in patients with recurrent implantation failure (RIF) and RM patients than in fertile controls (Kwak-Kim et al. 2003; Ng et al. 2002) making it a new treatment target for RM patients. Winger et al. (2009) administered TNF- $\alpha$ inhibitors and ivIgGs in combination with low molecular weight heparin (LMWH) in RM patients, which elevated the live birth rate (LBR).

G-CSF is synthesized by immune and endometrial cells (Makinoda et al. 2008). Recently, a few studies indicated possible benefits of G-CSF administration in RIF and RM patients (Scarpellini and Sbracia 2009; Wurfel et al. 2010). Scarpellini and Sbracia (2009) investigated G-CSF administration in patients with idiopathic RM and reached a LBR of $82.8 \%$ in the treatment group compared to $48.5 \%$ in the control group.

Within our study, we investigated G-CSF application in RM patients undergoing assisted reproductive therapy (ART) and compared the LBR with RM patients receiving no medication or LMWH, Cortisone or ivIgG. The study population consisted of 127 patients and 78 controls undergoing 199 and 127 in vitro fertilisation/intracytoplasmic sperm injection (IVF/ICSI) cycles, respectively.

\section{Materials and Methods}

Since 2002, RM patients who underwent ART at the Kinderwunsch Centrum Munich (KCM, Germany) were offered G-CSF treatment. In total, 7,410 were treated at the KCM during this period, including 649 RM patients. All patients underwent IVF/ICSI. The data were evaluated according to the following inclusion and exclusion criteria: at least two early miscarriages (excluding RM patients with biochemical pregnancies), no maternal or paternal chromosomal aberrations, no ART with heterologous oocytes or sperms, no immunmodulatory treatments like TNF- $\alpha$ blocker or donor leukocytes. In addition, patients were all negative for relevant uterine anomalies, infections and endocrine dysfunctions (like congenital adrenal hyperplasia or Basedow disease). The thyroid-stimulating hormone (TSH) level of patients with Hashimoto thyroiditis was adjusted by thyroid hormones to values $<2.5 \mathrm{IU} / \mathrm{ml}$. In cases with coagulation disorders, like mutations in the factor $\mathrm{V}$ Leiden or prothrombin gene, LMWH was administered daily, patients with anti-phospholipid antibodies were treated with LMWH and acetylsalicylic acid (Aspirin).

According to the guidelines of the German Society for Gynecology and Obstetrics the following investigations 
were performed: anti-phospholipid antibodies, such as anti-cardiolipin antibodies, lupus-anticoagulants and $\beta 2$-glycoproteins. In patients strongly suspected of having anti-phospholipid syndrome with a negative test result non-established antiphospholipid-antibodies such as phophatidylserin as well as inositol and annexin $\mathrm{V}$ levels were tested. To exclude autoimmune thyroid disease we measured basal TSH and anti-TPO antibodies in all patients. In case of suspected intestinal diseases, such as celiac disease, we examined transglutaminase antibodies. In addition, we investigated NK cell levels and the Th1/Th2 ratio in the peripheral blood of all patients. Furthermore, we performed a crossmatch-test and HLA typing of both partners. Since 2006, killer-cell immunglobulin-like receptor (KIR)-typing, and more recently, regulatory $\mathrm{T}$ cells were additionally part of our routine set up.

Only 127 RM patients (199 treatment cycles) fulfilled the inclusion criteria. In total, $n=49$ patients received G-CSF, 11 patients $1 \times 34$ Mill IU per week and 38 patients $2 \times 13$ Mill IU G-CSF/week starting at the day of embryo transfer until the 12th week of pregnancy. The subgroup 1, $n=33$ patients $(n=46$ cycles $)$, did not receive any medication, the subgroup 2 consisted of $n=45$ patients ( $n=81$ cycles) with other medicaments like LMWH enoxaparin-natrium $40 \mathrm{mg}$ subcutaneously once daily, acetylsalicylic acid (100 mg), folic acid (5 mg) or Prednisone/Dexamethasone $(2.5-5.0 \mathrm{mg} / 0.5 \mathrm{mg})$ starting in the middle of the previous cycle until the evidence of an embryonic heart beat and Doxycycline $(1 \times 100 \mathrm{mg}$ for 5 days) beginning at the day of the embryo transfer. All study patients received folic acid $(0.5 \mathrm{mg})$ and progesterone vaginally $(3 \times 200 \mathrm{mg}$ in the luteal phase until 12th week of pregnancy).

All patients underwent ART, $80 \%$ with a long stimulation protocol and the others with an antagonist protocol. Patients with regular cycles started with gonadotropinreleasing hormone agonist on day 24 of the preceding cycle. Additionally, daily recombinant follicle-stimulating hormone (FSH) and/or human menopausal gonadotropin (HMG; 125-200 IU) injection were administered from the third day of the menstrual cycle. Patients were closely monitored (vaginal ultrasound, taking into account the number and medium size of follicles of both ovaries and the endometrial thickness as well as concentrations of estradiol, progesterone and luteinizing hormone). The FSH/ HMG dose was adjusted according to the individual ovarian response. Ovulation was induced with 10,000 IU hCG when at least three follicles $\geq 17 \mathrm{~mm}$ were present 36-38 h before oocyte pick up. Depending on the sperm parameters according to the WHO guidelines, an IVF/ICSI treatment was performed. The embryonal development was closely monitored and an ultrasound-guided embryo transfer was performed on day 1-3 (75.6\%), day $4(3.9 \%)$ or day $5(20.5 \%)$ after oocyte pick up.

Data were analyzed with SPSS for Windows, release 19.0 (SPSS; Chicago, IL, USA). Results are given as mean \pm standard deviation (minimum-maximum), unless stated otherwise. Differences between the groups were analysed by the non-parametric Mann-Whitney test and the $\chi^{2}$ test, $p<0.05$ were regarded as statistically significant.

\section{Results}

\section{Study Population}

Mean age was $37.3 \pm 4.4$ years $(26-45)$. Patients receiving G-CSF had undergone significantly more early $(p<0.001)$ and late miscarriages $(p<0.001)$ as compared to the subgroup 1 and 2. In general, $6.02 \pm 3.8(0-17)$ ART cycles were performed before G-CSF treatment administration. $8.9 \pm 5.1$ (1-23) oocytes were reached during oocyte pick up of which $5.2 \pm 3.5$ (1-13) could be fertilized. Most embryo transfers were performed with $2 \pm 0.6$ (1-3) embryos and embryo transfer took place at day 5 in $37 \%$ of the patients receiving G-CSF compared to $12 \%$ in the controls without any

Table 1 Study population

\begin{tabular}{lccc}
\hline & G-CSF $(N=49)$ & $\begin{array}{l}\text { No medication (subgroup 1) } \\
(N=33)\end{array}$ & $\begin{array}{l}\text { Other medication (subgroup 2) } \\
(N=45)\end{array}$ \\
\hline Age (years) & $37.63 \pm 3.97(27-44)$ & $37.61 \pm 4.41(26-44), p=0.73$ & $37.61 \pm 4.41(26-44), p=0.48$ \\
Number of cycles undergone before treatment & $6.53 \pm 3.99(1-16)$ & $5.27 \pm 2.98(0-12), p=0.22$ & $6.02 \pm 4.1(0-17), p=0.56$ \\
Number of oocytes & $9.41 \pm 5.59(1-23)$ & $9.58 \pm 5.04(1-23), p=0.65$ & $7.84 \pm 4.47(1-18), p=0.17$ \\
Number of fertilized oocytes & $5.69 \pm 3.92(1-13)$ & $4.97 \pm 3.04(1-12), p=0.78$ & $4.91 \pm 3.43(1-13), p=0.38$ \\
Number of transferred embryos & $2.14 \pm 0.68(1-3)$ & $2.45 \pm 0.67(1-3), p=0.04$ & $2.36 \pm 0.57(1-3), p=0.13$ \\
Number of early miscarriages (anamnestic) & $2.67 \pm 1.27(1-6)$ & $0.85 \pm 0.91(0-3), p<0.001$ & $0.64 \pm 0.74(0-3), p<0.001$ \\
Number of late miscarriages (anamnestic) & $0.2 \pm 0(0-1)$ & $1.82 \pm 1.04(0-4), p<0.001$ & $1.78 \pm 1.02(0-4), p<0.001$ \\
\hline
\end{tabular}

Values are given as mean \pm SD (minimum-maximum), patients receiving G-CSF or no medication at all (subgroup 1) or other medications like LMWH, ASS 100, folic acid, Prednisone/Dexamethasone or Doxycycline (subgroup 2) 
medication $(p=0.05)$ and $9 \%$ in the controls receiving additional medications ( $p=0.005)$ (Table 1$)$.

In total, $71.36 \%$ patients received good quality embryos, $73.61 \%$ with G-CSF treatment, $62.22 \%$ in subgroup 1 and $76.25 \%$ in subgroup 2 . Good quality of an embryo was defined as an adequate morphology on the specific day of transfer according to the most recently published Istanbul criteria (2011).

\section{G-CSF Treatment}

Significant differences were present between RM patients receiving G-CSF and subgroups without G-CSF considering pregnancy rate (PR) and LBR. A PR of $47 \%$ and a LBR of $32 \%$ were achieved after G-CSF administration. In comparison to the G-CSF group, the subgroup of RM patients with some other medication (subgroup 2; Fig. 1) showed a PR of $27 \%, p=0.016$, and a LBR of $14 \%$, $p=0.006$, while the subgroup with no medication reached

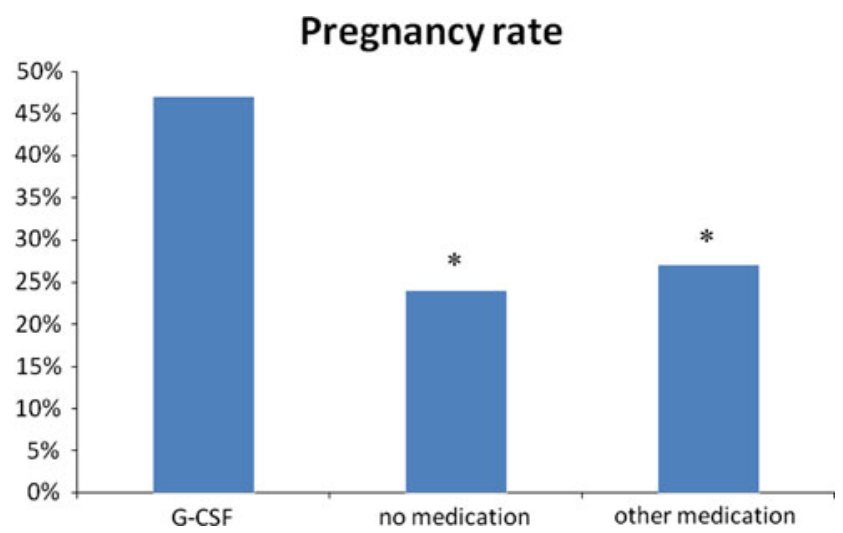

Fig. 1 Pregnancy rates of patients receiving either G-CSF, no medication at all (subgroup 1) or other medications like LMWH, ASS 100, folic acid, Prednisone/Dexamethasone or Doxycycline (subgroup 2). $* p<0.05$

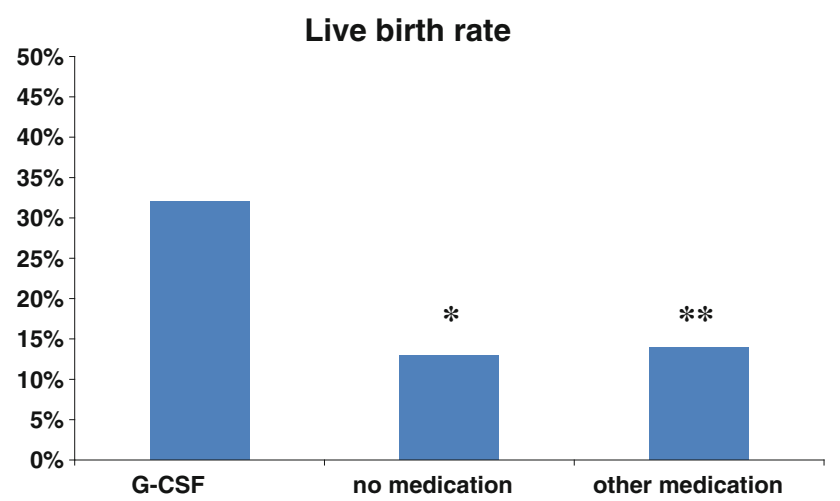

Fig. 2 Live birth rate of patients receiving either G-CSF, no medication at all (subgroup 1) or other medications like LMWH, ASS 100, folic acid, Prednisone/Dexamethasone or Doxycycline (subgroup 2). $* p<0.05 ; * * p<0.01$ a PR of $24 \%, p=0.016$, and a LBR of $13 \%, p=0.016$ (subgroup 1; Figs. 1 and 2).

Focussing on the subgroup of RM patients with G-CSF treatment, more patients receiving $1 \times 34$ Mill IU G-CSF (72\%) delivered a healthy baby compared to $21 \%$ patients receiving $2 \times 13$ Mill IU G-CSF, $p=0.001$.

Considering the occurrence of twin pregnancies, $4 \%$ (2/49) of patients receiving G-CSF delivered a multiple pregnancy, compared to $0 \%$ of patients without G-CSF (subgroup 2) and $6 \%$ (2/33) of patients without G-CSF or any other medication (subgroup 1).

Side effects were present in less than $10 \%$ of patients, consisting of irritation at the injection side, slight leukocytosis, rise in the temperature $\left(<38{ }^{\circ} \mathrm{C}\right)$, mild bone pain and hyperemesis gravidarum. None of the newborn showed any kind of malformations.

\section{Discussion}

Within our retrospective cohort study, PR and LBR of RM patients with G-CSF treatment were compared to patients receiving other relevant medications like LMWH and Aspirin, folic acid, Cortisone or Doxycyclin and a subgroup of patients without any medication. There were significant differences, concerning PR and LBR between the subgroups: a PR of $47 \%$ was achieved after G-CSF administration, of $27 \%$ in RM patients with alternative medications and $24 \%$ in cycles without medication.

However, we had some bias in our study: First of all, patients receiving G-CSF had significantly more often a day 5 embryo transfer as compared to the other patients. Of course, this "benefit" was counteracted due to the fact that G-CSF patients have undergone more early and late miscarriages, thus being a more severely affected RM subgroup. Another bias might be that the subgroup 2, which received a wide range of medical treatments for different indications, was a heterogeneous population. Thus, it is complicated to reconcile and compare these patients. This could be one of the reasons for the low rates of pregnancy success.

So far, there are no treatment options in patients with unexplained RM which are established and distinctly justified. According to a recent literature review, even the application of LMWH could not be proven to be completely safe and generally be of beneficial impact in patients undergoing ART (Bohlmann 2011).

By now, the only randomised controlled trial concerning G-CSF treatment in RM patients was the one by Scarpellini et al. (2009). Altogether, 68 women with primary unexplained RM, all with at least four consecutive miscarriages, were divided in a study $(n=35)$ and a placebo $(n=33)$ group. In contrast to our study, the patients were all 
younger than 39 years, did not undergo ART and received a G-CSF dosage which was $1 \mu \mathrm{g}(100,000 \mathrm{IU}) / \mathrm{kg} /$ day starting at the sixth day after ovulation. In addition, we did not adjust our G-CSF dosage due to patients weight and did not administer G-CSF daily. Taking an example of a $70 \mathrm{~kg}$ patient, the dosage per week of Scarpellini would be much higher than ours (49 Mill IUvs. 26, respectively, 34 Mill IU). Although our study population was slightly larger, we did not have the opportunity to form a placebo group considering the strict laws of the German Ethical committees. Scarpellini and Sbracia (2009) did not report on significant side effects of G-CSF application except a mild skin rash and slight leukocyte elevation which corresponds to our data.

G-CSF has multiple effects on the immune system like induction of the Th2 answer (Pan et al. 1995) and inhibition of NK cells (Schlahsa et al. 2011) and blood mononuclear cells (Kitabayashi et al. 1995; Sugita et al. 2003). Moreover, experiments on animals and cell lines have proven a protective impact on fetuses (Novales et al. 1993) and trophoblast cells (Marino and Roguin 2008).

Presuming that an immunologic disorder might be one of the main reasons for RM, these patients could benefit concerning the immunmodulatory functions of G-CSF. Furthermore, in vitro G-CSF application seems to be favourable on the decidualization process of endometrial stromal cells (Tanaka et al. 2000). However, its specific effects on the endometrium as well as the feto-maternal interphase are not fully understood yet.

As RM is a very heterogeneous pathology, the main problem which we are facing is to specify the subgroup of RM patients, which benefits from G-CSF treatment. This could be a surrogate immunological marker which we were not able to identify yet. Scarpellini and Sbracia (2009) only selected patients with idiopathic RM. Winger et al. (2009), who administered Humira, chose patients with a significant Th1/Th2 bias.

Our data suggest that RM patients might benefit from G-CSF treatment, but a placebo-controlled clinical trial including more RM patients is needed as well as the ongoing analysis of relevant immunological markers in order to further specify which RM patient will benefit from immunmodulatory treatments.

\section{References}

Alpha Scientists in Reproductive Medicine and ESHRE Special Interest Group of Embryology (2011) The Istanbul consensus workshop on embryo assessment: proceedings of an expert meeting. Hum Reprod 26:1270-1283

Bohlmann MK (2011) Effects and effectiveness of heparin in assisted reproduction. J Reprod Immunol 90:82-90
D'Addio F, Riella LV, Mfarrej BG et al (2011) The Link between the PDL1 costimulatory pathway and Th17 in fetomaternal tolerance. J Immunol 187:4530-4541

Faridi RM, Agrawal S (2011) Killer immunoglobulin-like receptors (KIRs) and HLA-C allorecognition patterns implicative of dominant activation of natural killer cells contribute to recurrent miscarriages. Hum Reprod 26:491-497

Hiby SE, Apps R, Sharkey AM et al (2010) Maternal activating KIRs protect against human reproductive failure mediated by fetal HLA-C2. J Clin Invest 120:4102-4110

Jin LP, Fan DX, Zhang T et al (2011) The costimulatory signal upregulation is associated with Th1 bias at the maternal-fetal interface in human miscarriage. Am $\mathbf{J}$ Reprod Immunol 66:270-278

Kitabayashi A, Hirokawa M, Hatano Y et al (1995) Granulocyte colony-stimulating factor downregulates allogeneic immune responses by posttranscriptional inhibition of tumor necrosis factor-alpha production. Blood 86:2220-2227

Kumar S, Naqvi RA, Khanna N et al (2011) Th3 Immune responses in the progression of leprosy via molecular cross-talks of TGF-beta, CTLA-4 and Cbl-b. Clin Immunol 141:133-142

Kwak-Kim JY, Chung-Bang HS, Ng SC et al (2003) Increased T helper 1 cytokine responses by circulating $\mathrm{T}$ cells are present in women with recurrent pregnancy losses and in infertile women with multiple implantation failures after IVF. Hum Reprod 18:767-773

Lee SK, Kim JY, Lee M et al (2012) Th17 and regulatory T cells in women with recurrent pregnancy loss. Am J Reprod Immunol 67:311-318

Makinoda S, Hirosaki N, Waseda T et al (2008) Granulocyte colonystimulating factor (G-CSF) in the mechanism of human ovulation and its clinical usefulness. Curr Med Chem 15:604-613

Marino VJ, Roguin LP (2008) The granulocyte colony stimulating factor (G-CSF) activates Jak/STAT and MAPK pathways in a trophoblastic cell line. J Cell Biochem 103:1512-1523

Mosmann TR, Coffman RL (1989) Heterogeneity of cytokine secretion patterns and functions of helper T cells. Adv Immunol 46:111-147

Ng SC, Gilman-Sachs A, Thaker P et al (2002) Expression of intracellular Th1 and Th2 cytokines in women with recurrent spontaneous abortion, implantation failures after IVF/ET or normal pregnancy. Am J Reprod Immunol 48:77-86

Novales JS, Salva AM, Modanlou HD et al (1993) Maternal administration of granulocyte colony-stimulating factor improves neonatal rat survival after a lethal group B streptococcal infection. Blood 81:923-927

Pan L, Delmonte J Jr, Jalonen CK et al (1995) Pretreatment of donor mice with granulocyte colony-stimulating factor polarizes donor $\mathrm{T}$ lymphocytes toward type- 2 cytokine production and reduces severity of experimental graft-versus-host disease. Blood $86: 4422-4429$

Practice Committee of tAmerican Society for Reproductive Medicine (2013) Definitions of infertility and recurrent pregnancy loss: a committee opinion. Fertil Steril 99(1):63 [Epub ahead of print]

Scarpellini F, Sbracia M (2009) Use of granulocyte colony-stimulating factor for the treatment of unexplained recurrent miscarriage: a randomised controlled trial. Hum Reprod 24:2703-2708

Schlahsa L, Jaimes Y, Blasczyk R et al (2011) Granulocyte-colonystimulatory factor: a strong inhibitor of natural killer cell function. Transfusion 51:293-305

Scholz C, Toth B, Santoso L et al (2008) Distribution and maturity of dendritic cells in diseases of insufficient placentation. Am J Reprod Immunol 60:238-245

Segerer SE, Rennemeier C, Kaemmerer U et al (2012) Dendritic cells: elegant arbiters in human reproduction. Curr Pharm Biotechnol 13:1378-1384 
Stirrat GM (1990) Recurrent miscarriage. Lancet 336:673-675

Sugita K, Hayakawa S, Karasaki-Suzuki M et al (2003) Granulocyte colony stimulation factor (G-CSF) suppresses interleukin (IL)-12 and/or IL-2 induced interferon (IFN)-gamma production and cytotoxicity of decidual mononuclear cells. Am J Reprod Immunol 50:83-89

Tanaka T, Miyama M, Masuda M et al (2000) Production and physiological function of granulocyte colony-stimulating factor in non-pregnant human endometrial stromal cells. Gynecol Endocrinol 14:399-404

Toth B, Haufe T, Scholz C et al (2010a) Placental interleukin-15 expression in recurrent miscarriage. Am $\mathrm{J}$ Reprod Immunol 64:402-410

Toth B, Jeschke U, Rogenhofer N et al (2010b) Recurrent miscarriage: current concepts in diagnosis and treatment. J Reprod Immunol 85:25-32

Wang WJ, Hao CF, Qu QL et al (2010) The deregulation of regulatory $\mathrm{T}$ cells on interleukin-17-producing $\mathrm{T}$ helper cells in patients with unexplained early recurrent miscarriage. Hum Reprod 25:2591-2596

Winger EE, Reed JL, Ashoush S et al (2009) Treatment with adalimumab (Humira) and intravenous immunoglobulin improves pregnancy rates in women undergoing IVF. Am J Reprod Immunol 61:113-120

Wurfel W, Santjohanser C, Hirv K et al (2010) High pregnancy rates with administration of granulocyte colony-stimulating factor in ART-patients with repetitive implantation failure and lacking killer-cell immunglobulin-like receptors. Hum Reprod $25: 2151-2152$

Yang KM, Ntrivalas E, Cho HJ et al (2010) Women with multiple implantation failures and recurrent pregnancy losses have increased peripheral blood $\mathrm{T}$ cell activation. Am J Reprod Immunol 63:370-378

Zhou J, Wang Z, Zhao X et al (2012) An increase of Treg cells in the peripheral blood is associated with a better in vitro fertilization treatment outcome. Am J Reprod Immunol 68:100-106 